\title{
Identification and Sensing Experiments with Fiber-Bragg-grating-based Spectral Labels in Free-space Optics System
}

\author{
Yao-Tang Chang, ${ }^{1}$ Bo-Rong Huang, ${ }^{2}$ Chih-Lun Shao, ${ }^{1}$ and Hsu-Chih Cheng ${ }^{2 *}$ \\ ${ }^{1}$ Kao Yuan University, Department of Information Technology \\ Kaohsiung 82151, Taiwan \\ ${ }^{2}$ National Formosa University, Department of Electro-Optical Engineering \\ No. 64, Wunhua Road, Huwei Township, Yunlin County 632, Taiwan
}

(Received February 4, 2017; accepted April 23, 2018)

Keywords: fiber Bragg grating (FBG), fiber laser, spectral label identification/sensing, optical code division multiple access (OCDMA), free-space optics (FSO) system

In this study, the spectral amplitude coding optical code division multiple access (SACOCDMA) is proposed for streaming data over a wireless transmission channel. A fiber-Bragggrating (FBG)-based parallel multiwavelength fiber laser is exploited as the light source, and a quasi-orthogonal $\mathrm{M}$-sequence code as the signature address code is prewritten in the FBGbased encoder to create an identifiable spectral label. In free-space optics (FSO) systems, the experimental implementation of the proposed scheme verified that spectral labels resulting from an object's signals could preserve clear sensing signals in the transmitted encoded spectral label. After multiple spectral-label signals were passed through a real free-space channel in air at normal atmospheric temperatures, the results of the identification and sensing experiment showed that the autocorrelation (matched) and cross-correlation (unmatched) scenarios were tested to identify an individual object using the spectral-label decoder, and then unmatched noise interference was completely cancelled to sense successfully the object's signal using a balanced photodetector.

\section{Introduction}

The basic principle of optical communications is to convert electronic signals into optical signals, and then covert the optical signals back into electric signals through media transmission. ${ }^{(1-3)}$ An optical fiber is generally adopted as the medium for transmission in traditional optical communications. The method of using air instead of an optical fiber as the medium for twoway information transmission of optical signals is called a free-space optic (FSO) system. ${ }^{(4-9)}$ Using invisible light waves rather than traditional radio waves as carrier waves, FSO technology can be used to transmit data, voices, and video without an optical fiber as well as to prevent collision and electromagnetic interference in identification and sensing applications using

*Corresponding author: e-mail: chenghc@nfu.edu.tw https://dx.doi.org/10.18494/SAM.2018.1935 
wireless optical communication instead of using radio frequency identification (RFID) in electromagnetic interference environments and scenarios such as airplanes, hospitals, and precision industries.

In current optical communication systems, increased network capacity and bandwidth are required as a result of the rapid development of information technology. Therefore, a new multitasking technology called spectral amplitude coding optical code division multiple access (SAC-OCDMA) has been proposed to act as an individual signature address code using a quasi-orthogonal spectral pattern. However, SAC-OCDMA schemes have not been experimented over free-space channels because the power attenuation resulting from wireless channel transmission and the cancellation of multiple access interference (MAI) have been investigated. ${ }^{(10-14)}$ Because an M-sequence codeword is characterized by quasi-orthogonality, the proposed SAC-OCDMA scheme using a fiber-Bragg-grating (FBG)-based fiber laser has high-capacity processing capabilities for nonsynchronized users and mitigates the problem of unmatched noise interference (i.e., leakage noise) when successfully implemented over FSO systems.

Fiber gratings are crucial in optical communication systems, and FBG is the most widely applied among the various types of fiber gratings. ${ }^{(15)}$ This system adopts a parallel multiwavelength fiber laser as the light source for encoders. ${ }^{(16,17)}$ In this study, spectral labels characterized by a unipolar maximum length sequence code (M-sequence code) were created using an FBG-based fiber laser and were assigned as signature address codewords to identify each object. The individual spectral label of an M-sequence pattern was also successfully sensed with balanced (i.e., original and complementary parts) FBG-based detection. In addition, the proposed FBG-based fiber laser configuration had immense power, high stability, and an independent temperature, and was capable of preventing mutual interference resulting from free-space channels. Compared with the previous configuration with a broadband light source in a wired fiber channel, ${ }^{(2,3)}$ a more stable and clearer spectral-label signal from an object can be obtained using an FBG-based spectral-label decoder with an M-sequence pattern and either data bit 1 or 0 can be retrieved by balanced detection.

\section{Experimental Principles and System Architecture}

Figure 1 shows the spectral-label encoding architecture of the proposed scheme in the current experiment. After a $980 \mathrm{~nm}$ pumped laser passed through an optical insulator, a $1 \times 2$ coupler, two FBGs of different wavelengths, a $3 \mathrm{~m}$ erbium-doped fiber, and a 50:50 half reflector were used to generate multiwavelength lasers of 1546.68, 1549.74, and $1552.36 \mathrm{~nm}$ as the light source for the encoding end of the experiment. Two optical signals with different coded wavelengths were transmitted using a $1 \times 2$ coupler connected to an electro/optic modulator (EOM). To achieve improved performance with respect to signal-to-noise ratio and optical identification, the M-sequence code, characterized by quasi-orthogonality, was prewritten in the FBG-based spectral-label encoder and decoder as the signature address code. For experimental design and implementation, as shown in Fig. 1, Objects \#1-2 were assigned and configured using the signature address codes for $\left[\begin{array}{lll}1 & 1 & 0\end{array}\right]$ and $\left[\begin{array}{lll}1 & 0 & 1\end{array}\right]$, respectively. Here, 


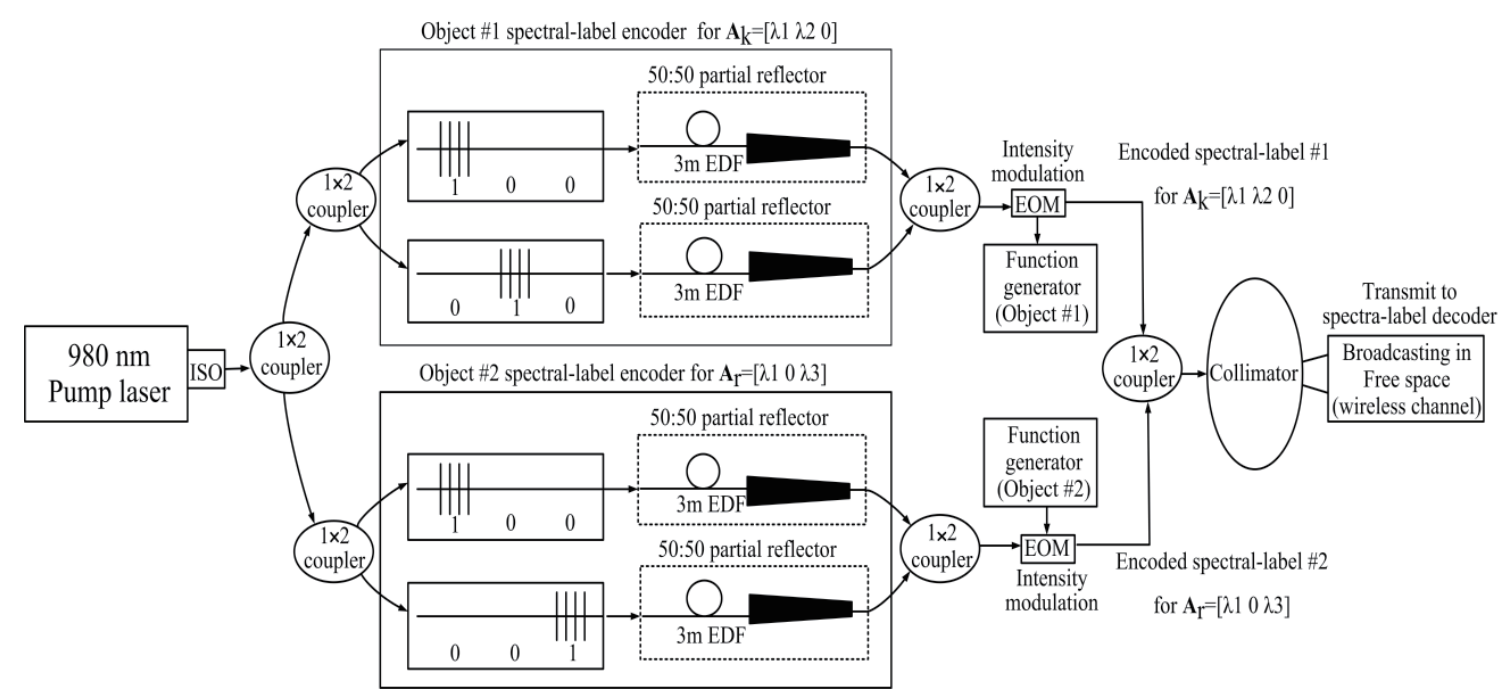

Fig. 1. Design and implementation of the proposed spectral-label encoding scheme.

the signature address codes of [ $\left[\begin{array}{lll}1 & 1 & 0\end{array}\right]$ and $\left[\begin{array}{lll}1 & 0 & 1\end{array}\right]$ with the M-sequence pattern for Objects \#1-2 corresponded to the spectral labels of $\left[\begin{array}{lll}\lambda_{1} & \lambda_{2} & 0\end{array}\right]$ and $\left[\begin{array}{lll}\lambda_{1} & 0 & \lambda_{3}\end{array}\right]$ as assigned by the FBG-based encoder, and the central wavelengths of the multiwavelength laser were set at $\lambda_{1}=1546.68, \lambda_{2}=$ 1549.74 , and $\lambda_{3}=1552.36 \mathrm{~nm}$ in the experiments.

Figure 2 illustrates the spectral-label decoding architecture of the proposed scheme in this experiment. The modulated spectral labels were transmitted and broadcast to the spectral-label decoder through the free space constituted by two collimators. The spectral labels were then exported from the spectral-label decoder to a balanced photodetector (BPD), and the spectral label received by Object \#1 was specifically designed for balanced detection by spectral-label decoder \#1. A BPD was adopted to conduct the correlation tests, unmatched noise interference from other users was deleted, and an oscilloscope was employed to correctly detect and sense the desired frequencies and signals of the spectral labels.

The spectral-label encoding configurations for Object $\# 1=\left[\begin{array}{lll}\lambda_{1} & \lambda_{2} & 0\end{array}\right]$ and Object $\# 2=\left[\begin{array}{lll}\lambda_{1} & 0 & \lambda_{3}\end{array}\right]$ are shown in Fig. 1. The FBG is prewritten in the M-sequence pattern $X_{k}$ and is expressed as

$$
X_{0}=\left[x_{0}, x_{1}, x_{2}, \ldots, x_{M-1}\right]
$$

where the M-sequence code pattern $X_{0}$ is $(0,1)$ sequences of length $M$, assigned as the spectrallabel codeword of Object \#1, such as the spectra-label codeword $\left[\begin{array}{lll}1 & 1 & 0\end{array}\right]$. According to the inherent cyclic property of an M-sequence, the M-sequence code pattern of Object \#k is expressed as

$$
X_{k}=T^{k-1} X_{0}=T^{k-1}\left[x_{0}, x_{1}, x_{2}, \ldots, x_{M-1}\right],
$$

where $T^{k-1}$ is the cyclic shifting operator, carried out cyclically to the right processing by $k-1$ places. In other words, the $k$-th Object's sequence $X_{k}$ can be obtained by cyclic right shifting 


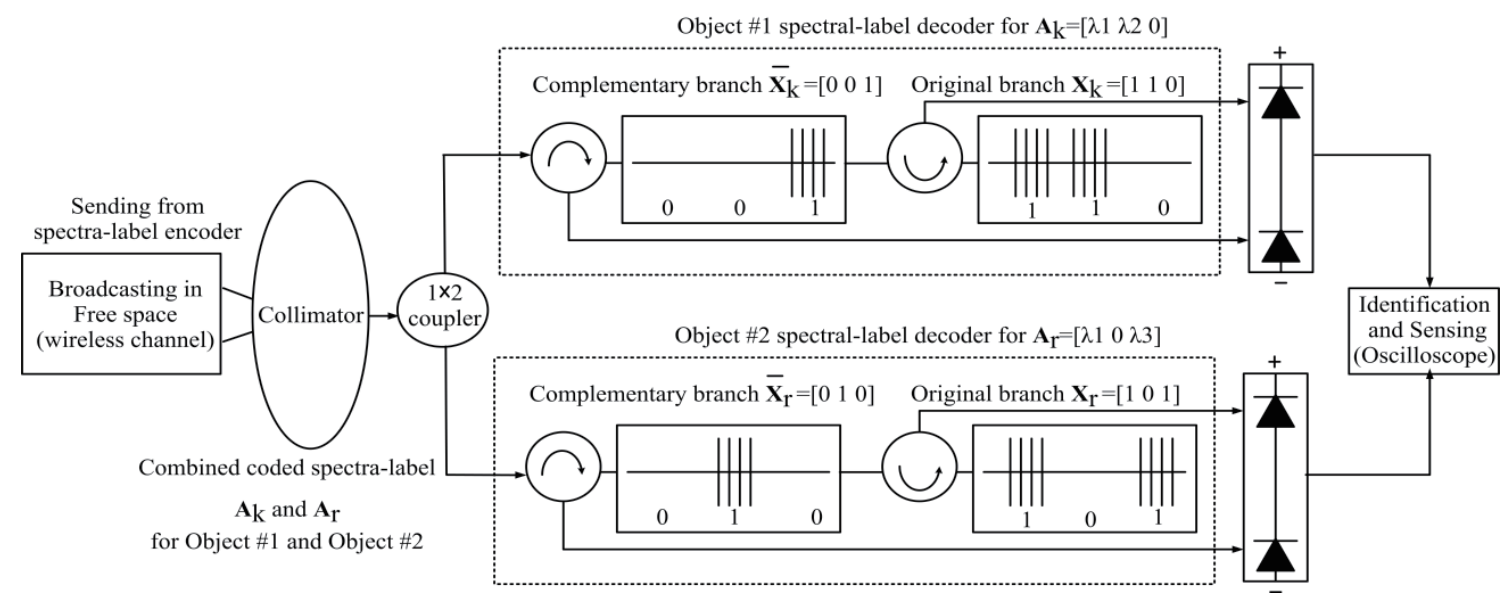

Fig. 2. Design and implementation of the proposed spectral-label decoding scheme.

$k-1$ processing based on the original sequence $X_{0}$. Following the simple encoding example

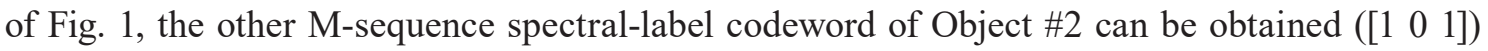
by cyclic right shifting two places from the original sequence, $\left[\begin{array}{lll}1 & 1 & 0\end{array}\right]$. In this experiment, the M-sequence code pattern of $X_{k}$ is mapped to the coded spectral label of $A_{k}$ in the wavelength domain and is expressed as

$$
\begin{gathered}
X_{k} \rightarrow A_{k}(\lambda), \\
A_{k}(\lambda)=T^{k-1} A_{0}(\lambda)=T^{k-1}\left[\lambda_{0}, \lambda_{1}, \lambda_{2}, \ldots, \lambda_{M-1}\right],
\end{gathered}
$$

where $A_{k}$ represents the spectral amplitude code of $X_{k}$. In this experimental design, Object encoders $\# 1$ and $\# 2$ are assigned as $\left[\lambda_{1} \lambda_{2} 0\right]$ and $\left[\lambda_{1} 0 \lambda_{3}\right]$, respectively, corresponding to [1546.68 $\mathrm{nm}, 1549.76 \mathrm{~nm}, 0]$ and [1546.68 $\mathrm{nm}, 0,1552.36 \mathrm{~nm}$ ] by adjusting the selected FBG components in the reflected wavelength $(1546.68,1549.76$, and $1552.36 \mathrm{~nm})$ to create the coded wavelengths of Objects \#1 and \#2.

For the quasi-orthogonal property of the proposed spectral label with an M-sequence for a signature address code, the basic principle of unmatched noise-interference cancellation, resulting from the use of balanced photodetection, is applied. ${ }^{(18)}$ The receiver end is designed with a reciprocal and symmetrical architecture, which is illustrated in Fig. 2. The proposed spectral-label decoders, consisting of an original and a complementary branch, are matched to the corresponding FBG-based encoders for Objects \#1 and \#2. The coded signal is broadcast in free space to arrive at the collimation of the received end. The receiver end is split into two branches by the $1 \times 2$ coupler. One branch is directed into the first upper branch of the FBGbased spectral-label encoder. This is equivalent to multiplying the coded signal by the spectrallabel codeword $A_{k}$, and hence a correlation value of $R^{(U)}{ }_{A A}$ is obtained in the upper photodiode. The second part of the signal is directed into the complementary FBG-based decoder and yields a correlation value of $R_{A \bar{A}}^{(L)}$ in the lower photodiode. The periodic cross-correlation between Objects $\# k$ and $\# r$ appearing in the upper branch is expressed as 


$$
R_{A A}^{(U)}(k, r)=\left[\sum_{i=0}^{M-1} a_{k}(i) \cdot a_{r}(i)\right]^{(U)} \begin{cases}\frac{M+1}{2}, & k=r \\ \frac{M+1}{4}, & k \neq r\end{cases}
$$

where $A(\lambda)=\left[a_{0}, a_{1}, a_{2}, \ldots, a_{M-1}\right]$ denotes a spectral label of length $M$ assigned as a codeword of Objects $\# k$ and $\# r$, depending on the different subscripts $k$ and $r$, respectively.

Similarly, the periodic cross-correlation between Objects $\# k$ and $\# r$ appearing in the lower branch is expressed as

$$
R_{A \bar{A}}^{(L)}(k, r)=\left[\sum_{i=0}^{M-1} a_{k}(i) \cdot \bar{a}_{r}(i)\right]^{(L)} \begin{cases}0, & k=r \\ \frac{M+1}{4}, & k \neq r\end{cases}
$$

where $\bar{A}(\lambda)=\left[a_{0}, a_{1}, a_{2}, \ldots, a_{M-1}\right]$ denotes the complementary spectral-label codeword of $A(\lambda)$.

The basic principle of the unmatched noise-interference cancellation effect, resulting from the use of a balanced photodetection approach in the proposed schemes, is that $\mathrm{M}$-sequence code families have a periodic cross-correlation between $A_{k}(\lambda)$ and $A_{r}(\lambda)$ in the upper branch, which is equal to the periodic cross-correlation between $A_{k}(\lambda)$ and $\bar{A}_{r}(\lambda)$ in the lower branch [i.e., $R_{A A}^{(U)}(k, r)=R_{A \bar{A}}^{(L)}(k, r)$, for $\left.k \neq r\right]$. A receiver computing $R_{A A}(k, r)-R_{A \bar{A}}(k, r)$ for User $\# k$ eliminates the influence of unmatched noise interference produced by the other users. Hence, a balanced detector for Object $\# k$ implements the correlation subtractions, as expressed in Eq. (6).

$$
R_{A A}^{(U)}(k, r)-R_{A \bar{A}}^{(L)}(k, r) \begin{cases}\frac{M+1}{2}, & k=r \\ 0, & k \neq r\end{cases}
$$

Following the subtraction of $R_{A \bar{A}}(k, r)$ from $R_{A A}(k, r)$ in the balanced photodiode, the data bits of Object \#k are sensed and extracted.

To investigate the unmatched noise-interference cancellation and to ensure reliable transmission over FSO systems, the autocorrelation and cross-correlation are measured from decoders of Objects $\# 1$ and $\# 2$ to verify the unmatched noise-interference cancellation when only the coded spectral Object \#1 for $A_{k}=\left[\lambda_{1} \lambda_{2} 0\right]$ was transmitted. Hence, the desired encoded spectral label for $\left[\lambda_{1} \lambda_{2} 0\right]$ may be monitored and measured before and after modulation by the EOM in this experimental design. Different from the previous scheme, which was applied only in a wired environment, ${ }^{(13-15)}$ the proposed scheme was tested to ensure reliable transmission over free space at normal atmospheric temperatures. The transmission distance in this experimental setup was about $2 \mathrm{~m}$.

To pass through a real air gap of a free space channel in air at atmospheric temperatures, two scenarios of matched and unmatched spectral-label codewords are evaluated to measure 
the results from the sensing decoder in the spectral powers of matched $\left[\lambda_{1} \lambda_{2} 0\right]$ and unmatched $\left[\begin{array}{lll}\lambda_{1} & 0 & \lambda_{3}\end{array}\right]$. In this experiment, the Object \#1 spectral-label encoder, configured by $\left[\begin{array}{lll}\lambda_{1} & \lambda_{2} & 0\end{array}\right]$, transmitted only an analog signal of $1 \mathrm{MHz}$ frequency, after which the autocorrelation and cross-correlation values were measured individually on the spectral-label decoder of Object $\# 1 X_{k}=\left[\begin{array}{lll}1 & 1 & 0\end{array}\right]$ and Object \#2 $X_{r}=\left[\begin{array}{lll}1 & 0 & 1\end{array}\right]$. The monitoring and measuring points of the experimental results are arranged and designed as shown in Fig. 3.

Figure 4 shows the the spectral label's spectrum of encoding pattern $X_{k}=\left[\begin{array}{lll}1 & 1 & 0\end{array}\right]$, configured before entering the EOM. In addition, Fig. 5 shows the spectrum of the object being modulated by the EOM and then entering the free-space channel.

The spectral range of the optical spectrum analyzer was regularly set at $1545-1555 \mathrm{~nm}$ with a resolution of $0.1 \mathrm{~nm}$. The spectral label's spectrum of encoding pattern generated

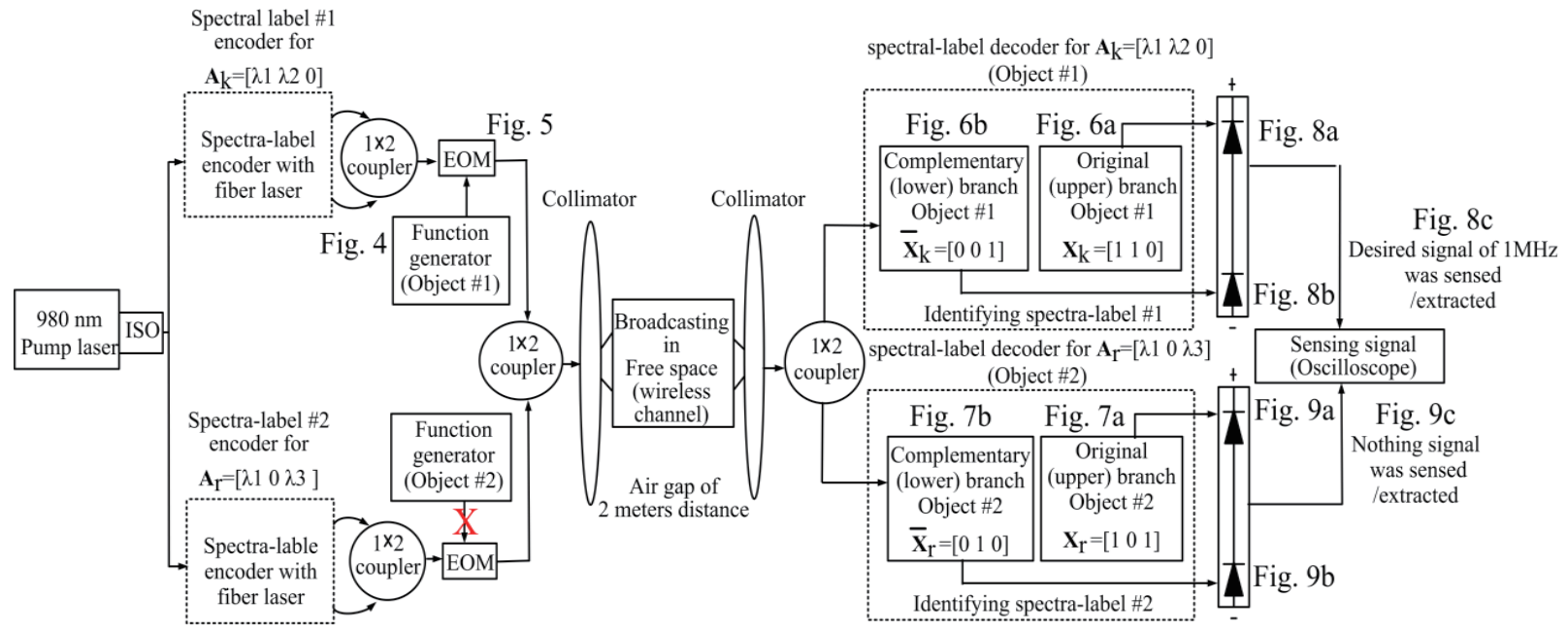

The auto-correlation and cross-correlation were measured to verify un-matched noise interference cancellation

Fig. 3. (Color online) Monitoring and measuring points for optical spectral-label experiments.

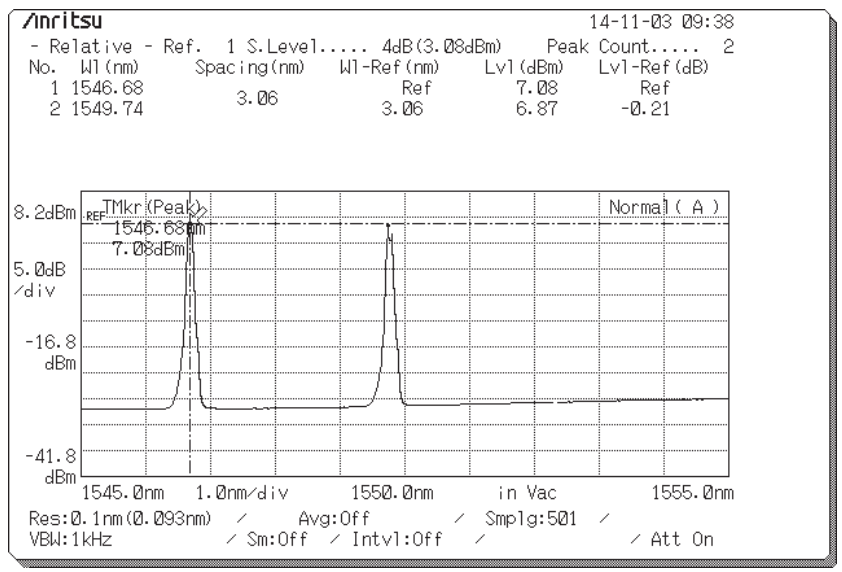

Fig. 4. Spectrum of the spectral label encoding [ $\left[\begin{array}{lll}1 & 1 & 0\end{array}\right]$ pattern before entering the EOM.

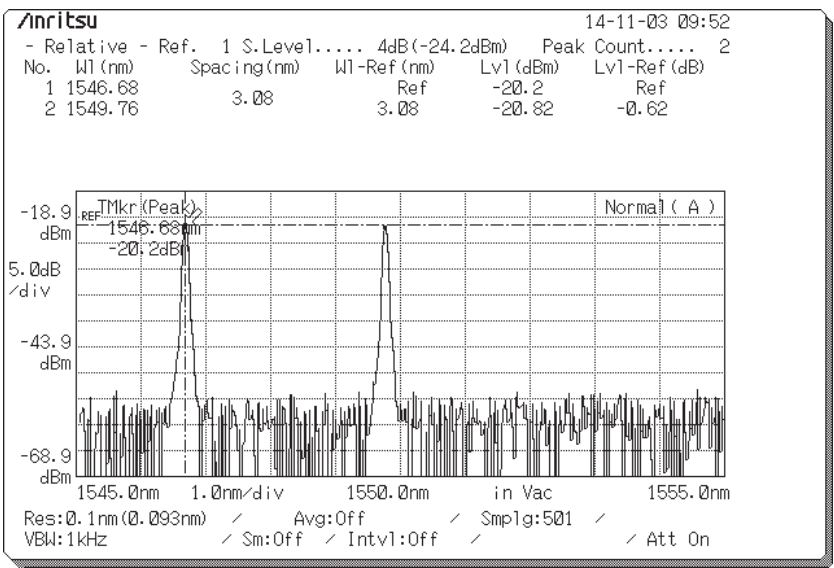

Fig. 5. Spectrum of the object being modulated by the EOM and then entering the 2-m-long free-space channel. 
optical signals with penetration wavelengths of $1546.68,1549.74$ (i.e., shifting to 1549.76 after EOM component), and $1552.36 \mathrm{~nm}$ in a practical experiment, which represented $\lambda_{1}, \lambda_{2}$, and $\lambda_{3}$, respectively. The present and absent wavelengths corresponded to binary data bits 1 and 0 , which were prewritten in the FBG-based encoder to create the reflected and transmitted wavelengths, respectively. Therefore, the SAC-based spectral-label codeword obtained in this study for Object \#1 was $\left[\begin{array}{lll}1 & 1 & 0\end{array}\right]$. To prove that the OCDMA system developed in this study effectively eliminated unmatched noise interference from other objects and correctly identified/ sensed the desired object signal, we used a spectral-label decoder, $X_{k}=\left[\begin{array}{lll}1 & 1 & 0\end{array}\right]$, which was matched with the spectral-label encoder and another spectral-label decoder of object $X_{r}=\left[\begin{array}{lll}1 & 0 & 1\end{array}\right]$, which was not matched and identified with the spectral-label encoder of object $X_{k}=\left[\begin{array}{lll}1 & 1 & 0\end{array}\right]$, for verification. When only the spectral-label encoding of Object \#1 was transmitted, the spectrallabel decoders of Objects \#1 and \#2 were individually used to measure the autocorrelation and cross-correlation results, respectively, to prove that the unmatched noise interference was cancelled.

Figure 6(a) shows the upper branch spectrum obtained using spectral-label decoder \#1 $X_{k}=\left[\begin{array}{lll}1 & 1 & 0\end{array}\right]$, which was matched with the spectral-label encoder of Object \#1, prewritten with $X_{k}=\left[\begin{array}{lll}1 & 1 & 0\end{array}\right]$. The reflection wavelengths obtained were 1546.24 and $1549.32 \mathrm{~nm}$, and the SACbased spectral-label codeword was $\left[\begin{array}{lll}1 & 1 & 0\end{array}\right]$. No spectra were detected in the lower branch, and thus the SAC-based spectral-label codeword was [0 $\left[\begin{array}{lll}0 & 0\end{array}\right]$ [Fig. 6(b)].

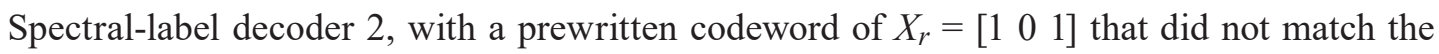
spectral-label encoder $\left[\begin{array}{lll}1 & 1 & 0\end{array}\right]$ of Object \#1, was adopted to obtain the upper branch reflection spectrum [Fig. 7(a)] with a reflection wavelength of $\lambda_{1}=1546.22 \mathrm{~nm}$ and the SAC-based spectral-label codeword of $\left[\begin{array}{lll}1 & 0 & 0\end{array}\right]$. The lower branch reflection spectrum is shown in Fig. 7(b), and a reflection wavelength of $\lambda_{2}=1549.30 \mathrm{~nm}$ and SAC-based spectral-label codeword of [ $\left[\begin{array}{lll}0 & 1 & 0\end{array}\right]$ were obtained. There are some differences between the spectral-label encoder and decoder wavelengths as a result of imperfections arising from the manufacturing processes of the FBGs.

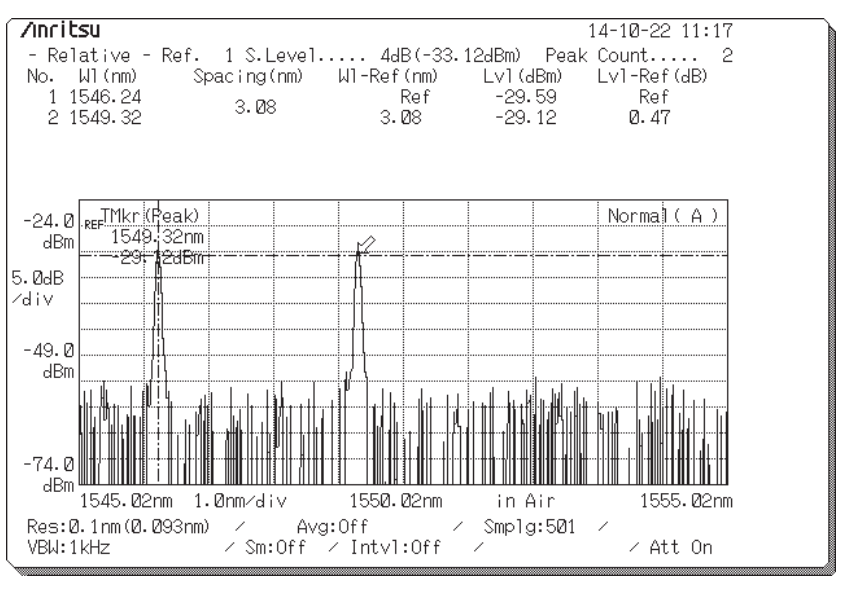

(a)

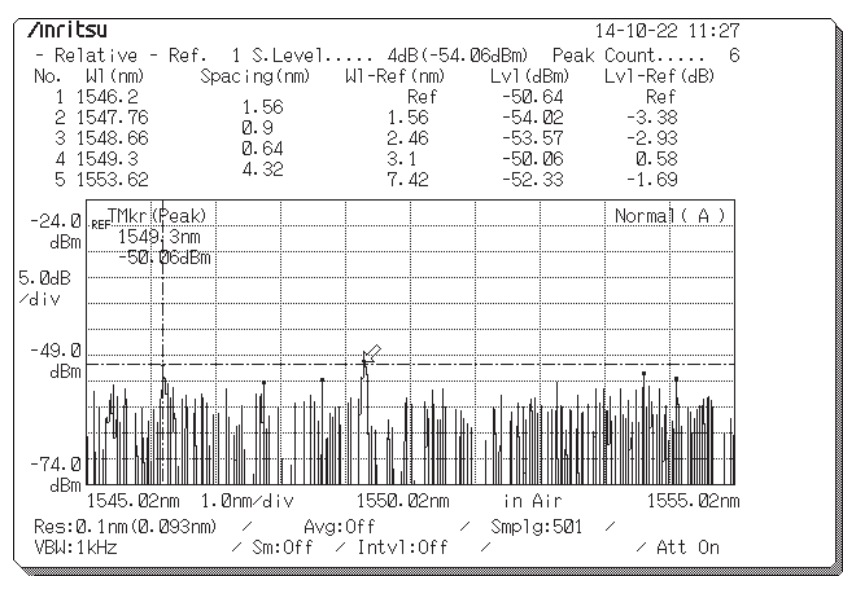

(b)

Fig. 6. Spectrum of spectral-label decoder \#1 on (a) upper branch and (b) lower branch. 


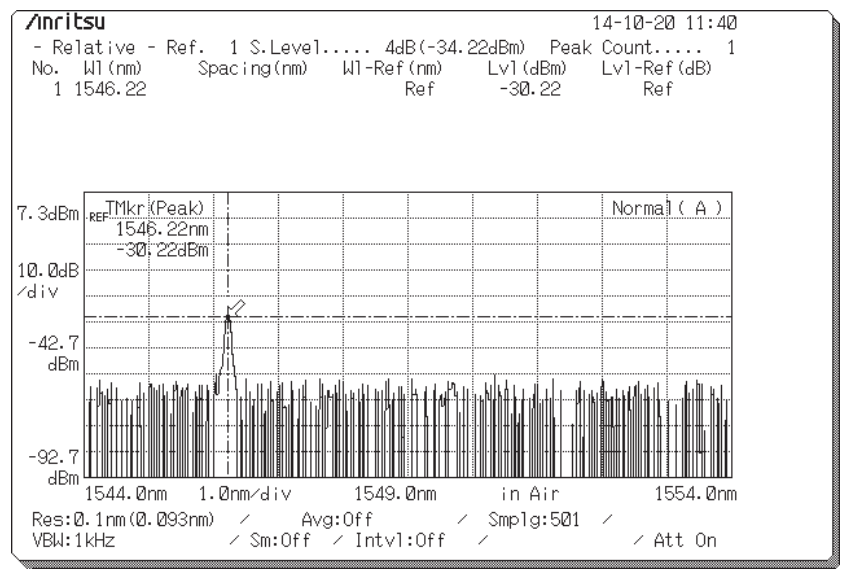

(a)

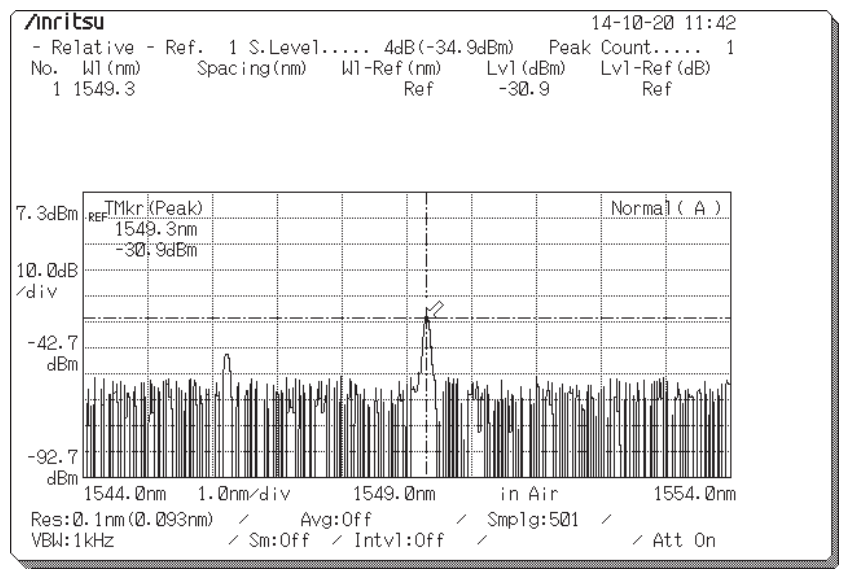

(b)

Fig. 7. Spectrum of spectral-label decoder \#2 on (a) upper branch and (b) lower branch.

\section{Results of Identification and Sensing and Discussion}

During this experiment, to extend the previous work of the authors on multiple access interference (MAI) cancellation over fiber-optic transmission lines, ${ }^{(10)}$ an analog object signal generator was used to provide $1 \mathrm{MHz}$ of analog electrical signals to the EOM. If the spectrallabel decoder matched the encoded spectral label it received, then the object frequencies inputted by the object into the EOM would be demodulated. However, if the spectral-label decoder did not match the spectral-label-encoded signals it received, then the BPD would not demodulate any object signals.

In the first experiment to measure the performance of autocorrelation over the free-space optical channel, the SAC spectral-label codeword [ [ $\left.\begin{array}{lll}1 & 1 & 0\end{array}\right]$ was entered and transmitted to a

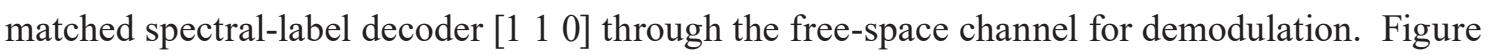
8(a) shows the BPD upper branch signals of spectral-label decoder \#1. Figure 8(b) presents the BPD lower branch signals of spectral-label decoder \#1; no signals were detected according to Eq. (5). Figure 8(c) illustrates the correlation subtraction of spectral-label decoder \#1 on the upper and lower branches of the BPD based on Eq. (6). This result showed that a signal of $-19 \mathrm{~dB}$ was detected at $1 \mathrm{MHz}$ via the BPD when the analog signal of Object \#1 was sensed and extracted.

In a second experiment to measure the performance of unmatched noise-interference cancellation (cross-correlation) over the free-space channel, SAC-based spectral-label codewords, $X_{k}=\left[\begin{array}{lll}1 & 1 & 0\end{array}\right]$, were entered and transmitted to an unmatched spectral-label decoder, $X_{r}=\left[\begin{array}{lll}1 & 0 & 1\end{array}\right]$, through the free-space channel for demodulation. Figure 9(a) presents the BPD upper branch signals of spectral-label decoder \#2, and a signal of $-34.6 \mathrm{~dB}$ was detected at 1 MHz. Figure 9(b) illustrates the BPD lower branch signals of spectral-label decoder \#2, and a signal of $-24.6 \mathrm{~dB}$ was detected at $1 \mathrm{MHz}$. Figure 9(c) shows the correlation subtraction of spectral-label decoder \#2 from the upper and lower branches of the BPD based on Eq. (6). This result showed that no signals were detected in the BPD when the analog signal of Object \#2 was sensed and extracted. 


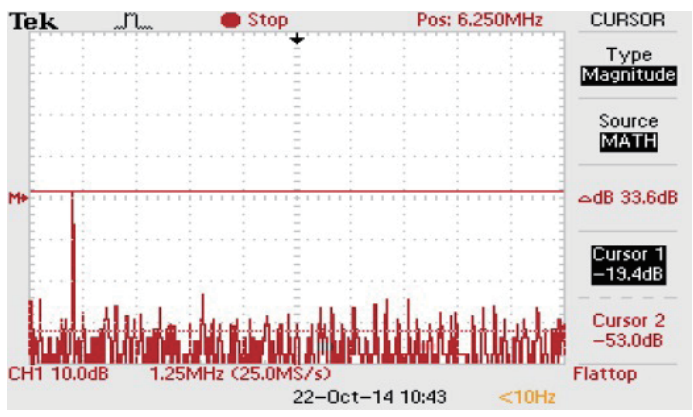

(a)

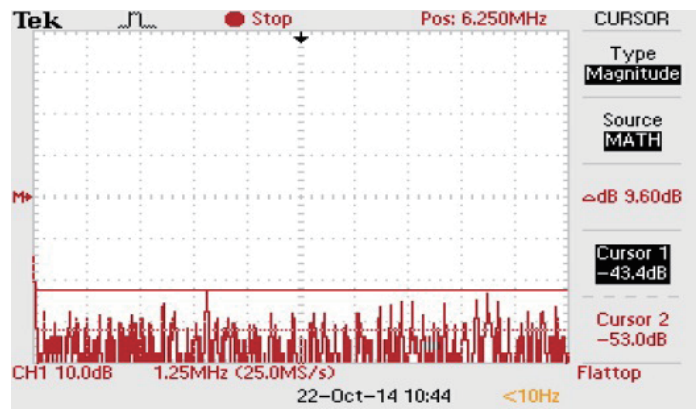

(b)

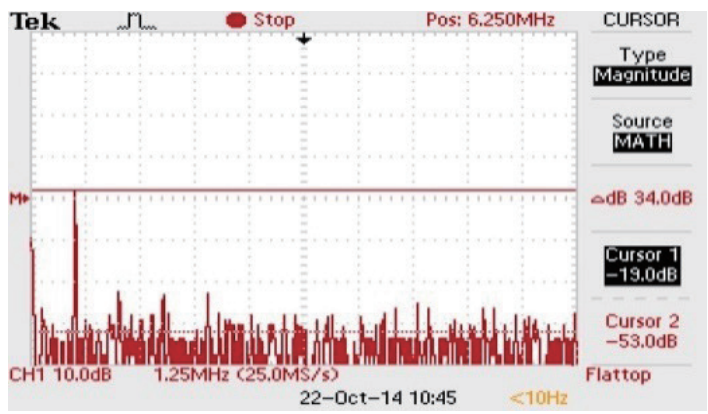

(c)

Fig. 8. (Color online) Signals of spectral-label decoder \#1 on (a) BPD upper branch, (b) BPD lower branch, and (c) BPD correlation subtraction of upper and lower branches to obtain the analog object's signal of $1 \mathrm{MHz}$.

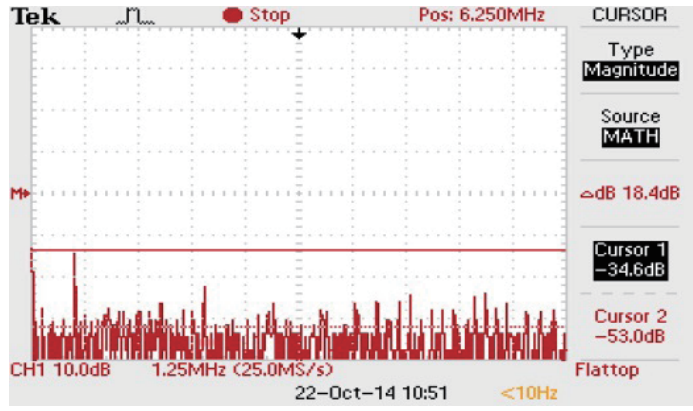

(a)

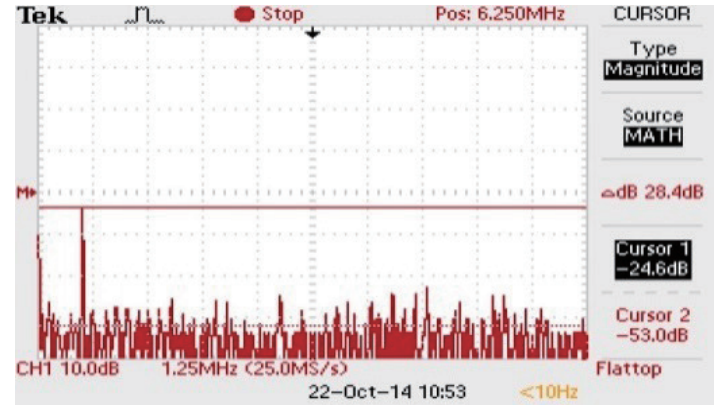

(b)

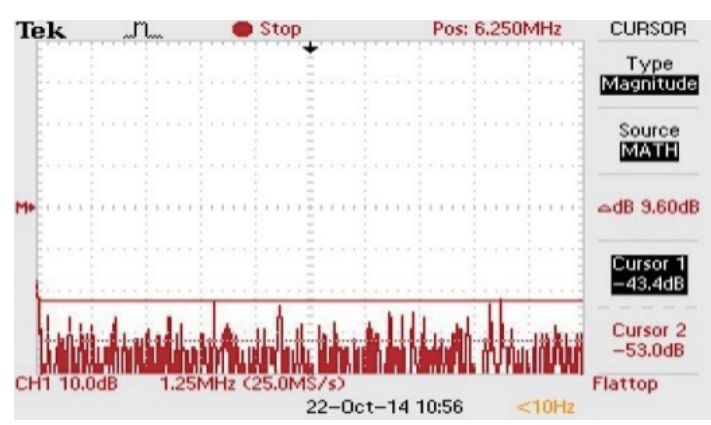

(c)

Fig. 9. (Color online) Signals of spectral-label decoder \#2 on (a) BPD upper branch, (b) BPD lower branch, and (c) BPD correlation subtraction of upper and lower branches yielding no signal. 
With the FBG-based parallel multiwavelength fiber laser as the light source and the quasiorthogonal M-sequence code as the signature address code of the spectral label prewritten in the FBG-based encoder, the results showed that an encoded spectral label was modulated by the user's electrical intensity using an EOM and then broadcast into the free space at the transmitter end. On the receiving end, the original and complementary FBG-based spectral-label decoder prewritten in the M-sequence code pattern was used to filter out the encoded spectral label of the desired object. Subsequently, the balanced direct detection was configured to retrieve the electrical signal of the desired object.

The BPD subtracted the signals of the upper and lower branches and converted the spectral labels into electric signals; then it successfully retrieved the object's sinusoidal waveform of the input frequency of the correct simulation signal. These results showed that this transmission system retrieved the correct object signal and was not influenced by unmatched noise interference, so even if the spectral-label codeword of the object was the same, the object's signal was demodulated without interference.

\section{Conclusions}

In this study, we adopted the architecture of SAC-OCDMA to identify and sense the frequencies of an object's signal. By using an FBG-based parallel multiwavelength fiber laser as the light source, each object had its own spectral label as a signature address code, and the spectral-label encoders and decoders of the objects were designed using an FBG component prewritten with the M-sequence pattern. In this experiment, the unique spectral label of an individual object provided a means of identification from various objects. The matched spectral-label decoder was configured to sense the desired object's signal. After the multiple spectral-label signals were passed through a real free-space channel $2 \mathrm{~m}$ in length, the autocorrelation and cross-correlation power values were obtained using the spectrallabel decoder with the M-sequence code pattern and then data bits 1 and 0 were retrieved by the balanced photodetector. This experiment showed that unmatched noise interference was completely cancelled to successfully sense the object's signal.

\section{Acknowledgments}

This work was supported in part by the Ministry of Science and Technology, Taiwan, under the grant nos. MOST 104-2221-E-150-043, MOST 105-2221-E-244-004, and MOST 106-2221E-244-002.

\section{References}

1 K. Prabu, S. Bose, and D. S. Kumar: Proc. 2012 Annu. IEEE India Conf. (INDICON 2012) 1176.

2 Y. T. Chang, Y. C. Lin, Y. C. Chen, and W. S. Wang: Multimedia Tools Appl. 76 (2017) 25421.

3 Y. T. Chang, H. C. Cheng, Y. C. Lin, and Y. X. Zheng: Proc. Eng. Technol. Innovation 5 (2017) 7.

4 C. Y. Chen, H. H. Lu, Y. P. Lin, P. Y. Wu, K. H. Wu, and W. Y. Yaug: Opt. Express 21 (2013) 23655.

5 N. Garg and S. Kumar: Proc. 2013 4th Int. Conf. Computing, Communications and Networking Technologies (ICCCNT 2013) 1-5.

6 H. L. Minh, Y. L. Yu, S. K. Liaw, H. Y. Hsu, W. C. Lu, and Z. Ghassemlooy: Proc. 2013 2nd Int. Workshop Optical Wireless Communications (IWOW 2013) 128. 
7 G. Nykolak, P. F. Szajowski, G. Tourgee, and H. Presby: Electron. Lett. 35 (1999) 578.

8 C. T. Yen and C. Y. Liu: Proc. Eng. Technol. Innovation 3 (2016) 1.

9 Y. T. Chang, C. L. Shao, B. R. Huang, and H. C. Cheng: Proc. 2015 Int. Workshop on Fiber Optics in Access Network (FOAN 2015) 17.

10 H. C. Cheng, C. H. Wu, C. C. Yang, and Y. T. Chang: IEEE Sens. J. 11 (2011) 2518.

11 I. Andonovic, L. Tancevski, M. Shabeer, and L. Bazgaloski: J. Lightwave Technol. 12 (1994) 1073.

12 C. F. Lam, D. T. K. Tong, M. C. Wu, and E. Yablonovitvh: IEEE Photonics Technol. Lett. 10 (1998) 1504.

13 J. F. Huang and D. Z. Hsu: IEEE Photonics Technol. Lett. 12 (2000) 1252.

14 C. C. Yang, J. F. Huang, and S. P. Tseng: IEEE Photonics Technol. Lett. 16 (2004) 641.

15 K. O. Hill and G. Meltz: J. Lightwave Technol. 15 (1997) 1263.

16 S. K. Liaw, H. Y. Tseng, and S. Chi: IEEE Photonics Technol. Lett. 12 (2000) 19.

17 A. Cucinotta, S. Selleri, L. Vincetti, and M. Zoboli: Opt. Commun. 156 (1998) 264.

18 Y. T. Chang and Y. C. Lin: Multimedia Tools Appl. 75 (2016) 9837.

\section{About the Authors}

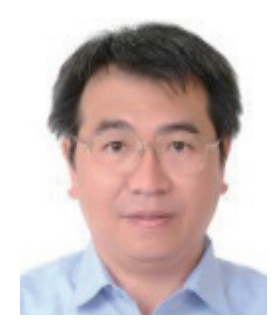

Yao-Tang Chang received his Ph.D. degree from the Department of Electrical Engineering at National Cheng Kung University, Taiwan, in 2007. He is currently an associate professor in the Department of Information Technology, Kao Yuan University, Taiwan. Before 2007, he served in the governmental communications department for over 20 years. His major research interests are in optical channel encryption/decryption and optical sensing and monitoring (t10066@cc.kyu.edu.tw).

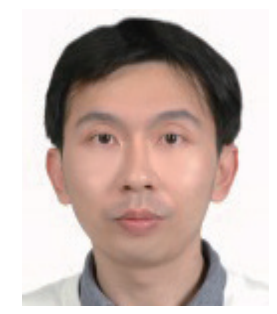

Bo-Rong Huang received his M.S. degree from the Department of ElectroOptical Engineering, National Formosa University, Taiwan. His major research interests are in the areas of multiuser optical communications, optical secure communication systems, and optical networks.

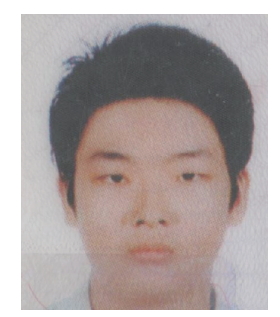

Chih-Lun Shao received his B.S. degree from the Department of Information Technology, Kao Yuan University, Taiwan. His major research interests are in the application of radio frequency identification.

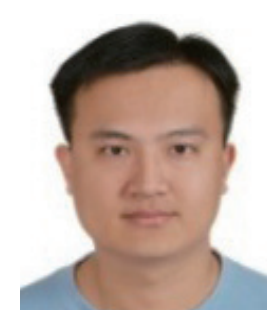

Hsu-Chih Cheng received his M.S. and Ph.D. degrees in electrical engineering from National Cheng Kung University, Tainan, Taiwan, in 2002 and 2006, respectively. He is currently a professor in the Department of Electro-Optical Engineering, National Formosa University, Taiwan. His major research interests lie in DWDM networking devices, optical systems design, and optic fiber sensors (chenghc@nfu.edu.tw). 